\title{
Home monitoring of physiology and symptoms to detect interstitial lung disease exacerbations and progression: a systematic review
}

\author{
Malik A. Althobiani (1) 1,2 , Rebecca A. Evans ${ }^{3}$, Jaber S. Alqahtani $\mathbb{1}^{1,4}$, Abdulelah M. Aldhahir ${ }^{5}$, \\ Anne-Marie Russell $\mathbb{1}^{6,7}$, John R. Hurst $\mathbb{1}^{1,7}$ and Joanna C. Porter $\mathbb{1}^{1,7}$
}

${ }^{1}$ UCL Respiratory, University College London, London, UK. ${ }^{2}$ Dept of Respiratory Therapy, Faculty of Medical Rehabilitation Sciences, King Abdulaziz University, Jeddah, Saudi Arabia. ${ }^{3}$ University College London Hospitals NHS Foundation Trust, London, UK. ${ }^{4}$ Dept of Respiratory Care, Prince Sultan Military College of Health Sciences, Dammam, Saudi Arabia. ${ }^{5}$ Respiratory Care Dept, Faculty of Applied Medical Sciences, Jazan University, Jazan, Saudi Arabia. ${ }^{6}$ University of Exeter College of Medicine and Health, Exeter, UK. ${ }^{7}$ These authors contributed equally.

Corresponding author: Joanna C. Porter (joanna.porter@ucl.ac.uk)

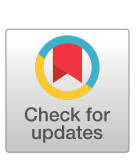

Copyright @The authors 2021

This version is distributed under the terms of the Creative Commons Attribution NonCommercial Licence 4.0. For commercial reproduction rights and permissions contact permissions@ersnet.org

This article has supplementary material available from openres.ersjournals.com

Received: 1 July 2021 Accepted: 27 Sept 2021

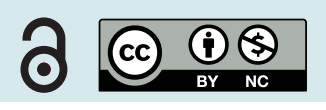

Shareable abstract (@ERSpublications)

First systematic review that provides supportive evidence for the feasibility and utility of home monitoring in ILD; further studies are necessary to evaluate approaches to detect exacerbation and/or progression https://bit.ly/2Y8OCJL

Cite this article as: Althobiani MA, Evans RA, Alqahtani JS, et al. Home monitoring of physiology and symptoms to detect interstitial lung disease exacerbations and progression: a systematic review. ERJ Open Res 2021; 7: 00441-2021 [DOI: 10.1183/23120541.00441-2021].

\section{Abstract}

Background Acute exacerbations (AEs) and disease progression in interstitial lung disease (ILD) pose important challenges to clinicians and patients. AEs of ILD are variable in presentation but may result in rapid progression of ILD, respiratory failure and death. However, in many cases AEs of ILD may go unrecognised so that their true impact and response to therapy is unknown. The potential for home monitoring to facilitate early, and accurate, identification of AE and/or ILD progression has gained interest. With increasing evidence available, there is a need for a systematic review on home monitoring of patients with ILD to summarise the existing data. The aim of this review was to systematically evaluate the evidence for use of home monitoring for early detection of exacerbations and/or progression of ILD.

Method We searched Ovid-EMBASE, MEDLINE and CINAHL using Medical Subject Headings (MeSH) terms in accordance with the PRISMA guidelines (PROSPERO registration number CRD42020215166).

Results 13 studies involving 968 patients have demonstrated that home monitoring is feasible and of potential benefit in patients with ILD. Nine studies reported that mean adherence to home monitoring was $>75 \%$, and where spirometry was performed there was a significant correlation $(r=0.72-0.98, p<0.001$ ) between home and hospital-based readings. Two studies suggested that home monitoring of forced vital capacity might facilitate detection of progression in idiopathic pulmonary fibrosis.

Conclusion Despite the fact that individual studies in this systematic review provide supportive evidence suggesting the feasibility and utility of home monitoring in ILD, further studies are necessary to quantify the potential of home monitoring to detect disease progression and/or AEs.

\section{Introduction}

Interstitial lung disease (ILD) is a general term for approximately 200 different diseases that may result in inflammation and scarring of the lung [1]. ILD is characterised by progressive dyspnoea, inflammation, fibrosis and reduced quality of life [1]. Most cases of ILD result from an aetiological factor, such as exposure to allergens, toxins or drugs or from an underlying autoimmune disease, with a modifying influence of genetics and exogenous factors such as air pollution [2-4]. In many cases the aetiology is unclear [5]. The most severe form of ILD is idiopathic pulmonary fibrosis (IPF) [1, 6], for which there is no cure [2]. The median survival time after diagnosis of untreated IPF is $2-5$ years [2, 7, 8]. The considerable variability seen between patients makes individual outcome prediction difficult. In addition, 
there is a lack of validated biomarkers of disease progression [6, 9]. Forced vital capacity (FVC) is one possible biomarker of disease progression and is usually measured intermittently. There are currently two anti-fibrotic drugs approved for use in IPF, nintedanib and pirfenidone, which have been demonstrated to slow the rate of FVC decline [10-13] and may increase median survival [10, 12, 14]. There is an urgent unmet need for better treatments. Currently all clinical trials of novel therapies for IPF have used the established end-point of rate of decline in FVC and have required large patient cohorts followed for a significant length of time (years) to identify meaningful treatment responses [15, 16]. More frequent measures, such as the use of home spirometry, may reduce the size, length of time and cost of clinical trials [17-20]. Ineffective drug treatments could also be identified at an earlier stage. More regular monitoring of physiological parameters in ILD might be of benefit in clinical practice and in research.

Patients with ILD may experience acute deteriorations (“exacerbations” or "acute exacerbations” (AEs)) of their condition, and there is a growing body of research into the detection of exacerbation in ILD [21, 22]. AEs of ILD (AE-ILD) are highly variable but may result in rapid respiratory deterioration, alveolar abnormalities, and in severe cases death [21-23]. This rapid progression can cause severe distress to patients and burden healthcare systems. The potential for home monitoring to identify progression, including AE, at an early stage is of significant interest. Supporting evidence exists in other respiratory diseases. It has been demonstrated that early detection of exacerbations in COPD speeds recovery time [24, 25]. Home monitoring has thus been recommended in lung diseases such as COPD to support earlier detection of exacerbation [26, 27]. It is suggested that similar technology may benefit patients with ILD [20, 28-32].

Another challenge in ILD is the marked inter-patient heterogeneity which makes it very difficult to accurately predict life expectancy and so to provide a reliable prognosis to individual patients and their families [22]. A benefit of home monitoring may be that more frequent monitoring of individual patients may allow prediction of a patient's personal trajectory that can inform prognostication and decisions of future care [31, 32].

The potential role for home monitoring in ILD has been amplified by the coronavirus disease 2019 (COVID-19) pandemic. The guidance from the British Lung Foundation and UK National Health Service for patients with ILD has been to "shield", to reduce the risk of contracting COVID-19 [33]. Home monitoring could arguably decrease the inherent risk in physical attendance at outpatient clinics [26, 30]. This may also prove useful beyond the COVID-19 pandemic, given that ILD can limit patient mobility and care is often centralised at tertiary centres [34, 35].

There is no existing systematic synthesis of the literature to examine the role of home monitoring to detect ILD exacerbation and/or disease progression. Thus, we aimed to systematically gather, summarise and evaluate the evidence not just for feasibility and reliability, but also on detection of AE-ILD and/or disease progression in this systematic review.

\section{Search methods}

\section{Protocol and registration}

We undertook a systematic review of the literature using a protocol in accordance with the preferred reporting items for systematic review and meta-analysis protocols (PRISMA-P 2015) [36, 37]. We prospectively registered this systematic review at PROSPERO (protocol registration number CRD42020215166).

\section{Eligibility criteria}

We utilised the PICO framework (participants/population, intervention(s)/exposure(s), comparator(s)/ control, main outcome(s)) as a search strategy. We systematically searched for studies on home monitoring and interstitial lung disease published worldwide with no restriction on date of publication. Home monitoring was defined as the regular use of any home-based technology and spirometry to monitor symptoms and/or physiological parameters (such as vital signs and spirometry) over a period of at least 3 weeks. The selected papers met the following inclusion criteria: 1) patients with confirmed diagnoses of ILD determined by the authors' local criteria; 2) written in English; 3) focus on home monitoring to detect exacerbations and/or progression in patients with ILD; 4) detected ILD exacerbations and progression; and 5) randomised controlled trials (RCTs), prospective or retrospective cohort observational studies or case-control studies. Studies were excluded if the following criteria were met: 1) studies that were conference abstracts, theses and book chapters; or 2) systematic reviews and meta-analyses (we screened the bibliography), literature reviews or qualitative studies. 


\section{TABLE 1 Use of Cochrane risk of bias tool to assess quality of randomised controlled trials}

\begin{tabular}{cccccccc} 
Study & $\begin{array}{c}\text { Random sequence } \\
\text { generation }\end{array}$ & $\begin{array}{c}\text { Allocation } \\
\text { concealment }\end{array}$ & $\begin{array}{c}\text { Selective } \\
\text { reporting }\end{array}$ & $\begin{array}{c}\text { Blinding subject } \\
\text { and personnel }\end{array}$ & $\begin{array}{c}\text { Blinding outcome } \\
\text { assessment }\end{array}$ & $\begin{array}{c}\text { Incomplete } \\
\text { outcome data }\end{array}$ & $\begin{array}{c}\text { Other source of } \\
\text { bias }\end{array}$ \\
\hline $\begin{array}{c}\text { MAHER et al. } \\
(2020)[38]\end{array}$ & Low & Low & Low & Low & Low & Low & Unclear \\
$\begin{array}{c}\text { MOOR et al. } \\
(2020)[30]\end{array}$ & Low & Low & Low & High & High & Low & Unclear \\
\hline
\end{tabular}

\section{Search strategy}

Between October and November 2020, we searched electronic databases for published articles at any date prior to this, and then updated the search in February 2021 to identify further relevant publications on ILD and home monitoring. We developed a search strategy with medical library staff and extensively searched the following databases: Ovid-EMBASE, Ovid-MEDLINE, Cumulative Index to Nursing and Allied Health Literature (supplementary tables S1 to S5). We also searched the references of studies thoroughly for any eligible articles. We searched the above electronic databases for Medical Subject Headings (MeSH) terms, and the main terms classified into three groups that describe ILD, home monitoring and progression. Further detailed related terms to this systematic review are included in supplementary tables S1 to S5.

\section{Data collection}

All studies found to be potentially eligible were retrieved from the electronic databases and stored for de-duplicating in the reference management software package EndNote. We exported the results, after removing duplicates, to the online software Rayyan, where the title and abstract of potential studies were screened by two independent reviewers (M.A. Althobiani and R.A. Evans). The software allows the two reviewers to include and exclude studies blindly, and when completed, disagreements were resolved by reading the full text and discussion. We exported the included studies to a new EndNote library, where the articles were read in full.

\section{Quality assessment}

Two authors (M.A. Althobiani and J.S. Alqahtani) conducted detailed quality assessment using the Cochrane risk of bias tool for the assessment of the included RCT studies, and the Newcastle-Ottawa tool was used in regard to the observational studies. The Cochrane risk of bias tool comprises seven domains. The Newcastle-Ottawa tool consists of three broad perspectives used to assess the quality of non-randomised studies included in this systematic review. The quality of the cohort studies is based upon a "star" system with a total possible score of nine stars. Study ratings are indicated with the following: 7-9 stars=good, 4-6 stars=fair, 1-3 stars=poor (tables 1 and 2).

\section{Synthesis of results}

Narrative synthesis was undertaken according to outcomes that were reported in the included studies with more emphasis given to studies of higher quality. We considered how differences in design, outcomes, intervention, population and setting may have contributed to any differences in observed results.

\section{Results}

The original search across five databases identified 1841 publications; 1533 articles remained after duplicates were manually reviewed and removed. A total of 1422 articles were excluded using title-only screening, followed by 79 exclusions after title and abstract screening. A total of 32 articles remained for full-text screening, 22 of which were excluded. Three articles were included as relevant from searching the references. Thus, 13 studies were considered for inclusion in this systematic review as depicted in the PRISMA flow diagram (figure 1).

\section{Description of the included studies}

11 prospective cohort studies and two RCTs were identified and included by the systematic search. The RCTs were conducted in the Netherlands and in multiple centres internationally. Seven cohort studies were conducted in the Netherlands, one in the UK, one in the USA, one in Germany, and one across the USA and Ireland.

\section{General description}

Characteristics of the included studies are summarised in table 3. The studies were published between 2016 and 2021 and involved a total of 968 recruited patients with ILD. The sample size for these studies ranged 


\begin{tabular}{|c|c|c|c|c|c|c|c|c|c|c|}
\hline Study & $\begin{array}{l}\text { Representativeness } \\
\text { of exposed cohort }\end{array}$ & $\begin{array}{l}\text { Selection of } \\
\text { non-exposed } \\
\text { cohort }\end{array}$ & $\begin{array}{l}\text { Ascertainment } \\
\text { of all-cause }\end{array}$ & $\begin{array}{l}\text { Outcome } \\
\text { not present } \\
\text { at the start } \\
\text { of study }\end{array}$ & $\begin{array}{l}\text { Comparability } \\
\text { of cohort }\end{array}$ & $\begin{array}{l}\text { Assessment } \\
\text { of outcome }\end{array}$ & $\begin{array}{c}\text { Adequate } \\
\text { follow-up } \\
\text { duration }\end{array}$ & $\begin{array}{c}\text { Adequate } \\
\text { follow-up } \\
\text { rate }\end{array}$ & Score & Quality \\
\hline $\begin{array}{l}\text { Russell et al. } \\
\qquad(2016) \text { [31] }\end{array}$ & 1 & 0 & 1 & 1 & 2 & 0 & 1 & 1 & 7 & Good \\
\hline $\begin{array}{l}\text { Johannson et al. } \\
\qquad(2017)[20]\end{array}$ & 1 & 0 & 1 & 1 & 1 & 1 & 1 & 1 & 7 & Good \\
\hline $\begin{array}{l}\text { Veit et al. (2020) } \\
\quad \text { [32] }\end{array}$ & 1 & 0 & 1 & 1 & 2 & 1 & 1 & 1 & 8 & Good \\
\hline $\begin{array}{l}\text { EDWARDS et al. } \\
\qquad(2020) \text { [39] }\end{array}$ & 0 & 0 & 1 & 1 & 2 & 1 & 1 & 1 & 7 & Good \\
\hline $\begin{array}{l}\text { Moor et al. } \\
\text { (2019) [45] }\end{array}$ & 1 & 0 & 1 & 1 & 0 & 1 & 1 & 1 & 6 & Fair \\
\hline $\begin{array}{l}\text { Moor et al. } \\
\qquad(2018)[40]\end{array}$ & 1 & 0 & 1 & 1 & 2 & 1 & 1 & 1 & 8 & Good \\
\hline $\begin{array}{l}\text { Moor et al. } \\
\text { (2020) [46] }\end{array}$ & 1 & 0 & 1 & 1 & 0 & 1 & 1 & 1 & 6 & Fair \\
\hline $\begin{array}{l}\text { Broos et al. } \\
\qquad(2017)[41]\end{array}$ & 1 & 0 & 1 & 1 & 2 & 1 & 1 & 1 & 8 & Good \\
\hline $\begin{array}{l}\text { MARCOUx et al. } \\
\text { (2019) [28] }\end{array}$ & 1 & 0 & 1 & 1 & 2 & 1 & 1 & 1 & 8 & Good \\
\hline $\begin{array}{l}\text { Noтн et al. } \\
\qquad(2021) \text { [43] }\end{array}$ & 1 & 1 & 1 & 1 & 2 & 1 & 1 & 1 & 9 & Good \\
\hline $\begin{array}{l}\text { Moor et al. } \\
\quad(2020)[42]\end{array}$ & 1 & 0 & 1 & 1 & 2 & 1 & 1 & 1 & 8 & Good \\
\hline
\end{tabular}

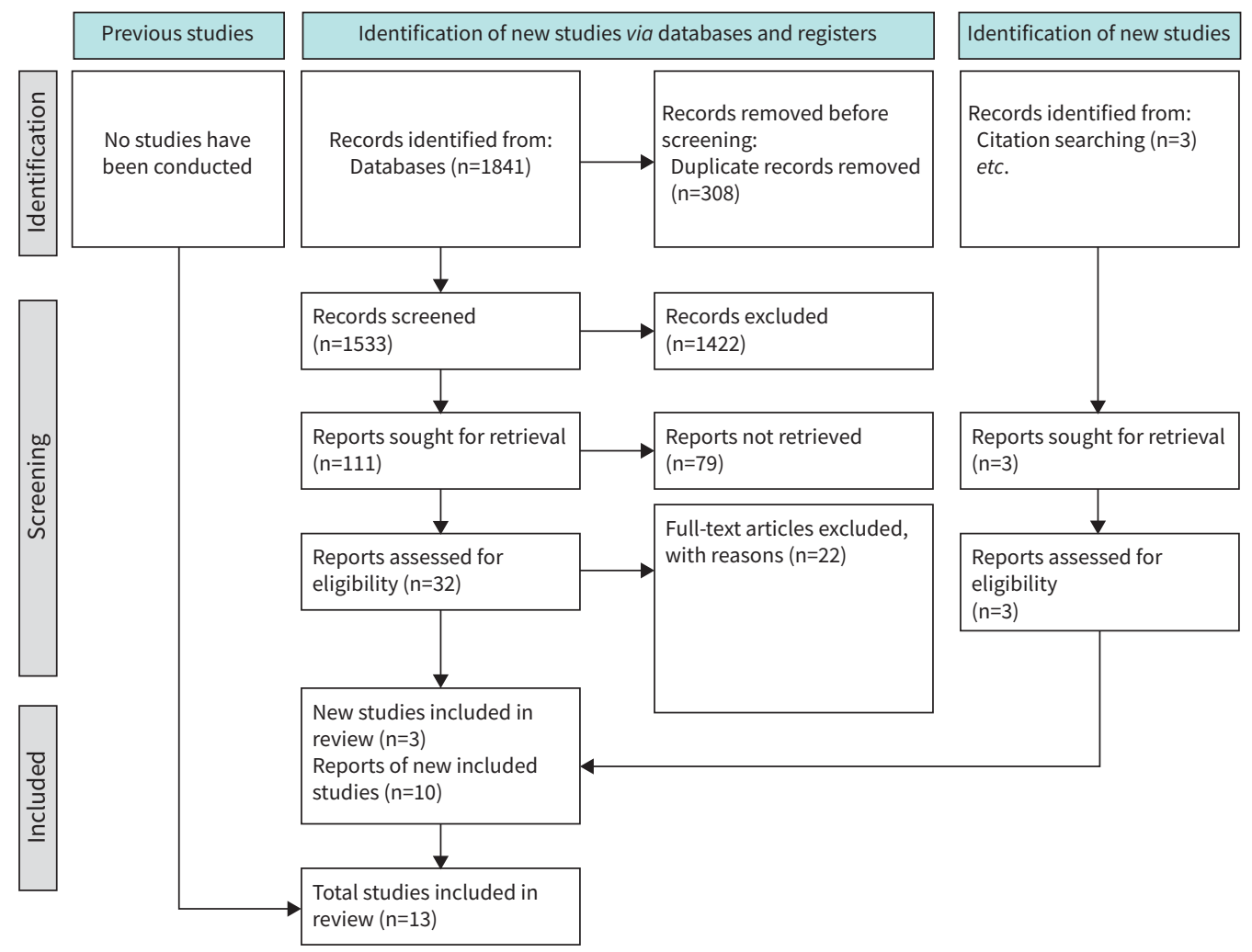


TABLE 3 Characteristics of included studies on home monitoring in interstitial lung disease (ILD) patients

\begin{tabular}{|c|c|c|c|c|c|c|c|c|c|}
\hline Study & $\begin{array}{l}\text { Setting/ } \\
\text { design }\end{array}$ & $\begin{array}{l}\text { Sample size and } \\
\text { characteristics }\end{array}$ & $\begin{array}{l}\text { Disease } \\
\text { group }\end{array}$ & $\begin{array}{l}\text { Clinic measures/ } \\
\text { frequency }\end{array}$ & $\begin{array}{l}\text { Home measures/ } \\
\text { frequency }\end{array}$ & $\begin{array}{l}\text { Study } \\
\text { length }\end{array}$ & Outcome & Quality & Results \\
\hline $\begin{array}{l}\text { Moor et al. } \\
\qquad(2020) \text { [30] }\end{array}$ & $\mathrm{NL} / \mathrm{RCT}$ & $\begin{array}{c}\mathrm{n}=90 \\
\text { Age }(\text { mean } \pm \mathrm{sD}): \\
71 \pm 6.9 \text { years } \\
\text { Intervention }(\mathrm{n}=46) \\
\text { Male: } 39(85 \%) \\
\text { Age }(\text { mean): } \\
70 \text { years } \\
\text { Control }(\mathrm{n}=44) \\
\text { Male: } 43(98 \%) \\
\text { Age (mean): } \\
72 \text { years }\end{array}$ & IPF & $\begin{array}{l}\text { Spirometry, K-BILD, } \\
\text { PESaM, EQ-5D-5L, } \\
\text { HADS, VAS, GRC, } \\
\text { EQ-VAS (baseline, } \\
\text { and at } 12 \text { and } \\
24 \text { weeks) }\end{array}$ & $\begin{array}{l}\text { FVC (Once daily) } \\
\text { K-BILD, PESaM, } \\
\text { EQ-5D-5L, HADS, } \\
\text { VAS, GRC, EQ-VAS } \\
\text { (weekly) }\end{array}$ & 24 weeks & $\begin{array}{l}\text { Investigate whether } \\
\text { a home monitoring } \\
\text { programme } \\
\text { improves HRQOL } \\
\text { and medication use } \\
\text { for patients with IPF }\end{array}$ & Moderate & $\begin{array}{l}\text { (1) Improved } \\
\text { psychological well-being } \\
\text { compared to standard } \\
\text { care alone (mean } \\
\text { difference } 1.04 \text { points; } \\
95 \% \mathrm{Cl} 0.09-2.00 ; \mathrm{p}=0.032) \\
\text { (2) Mean change in FVC } \\
\text { was not significantly } \\
\text { different between } \\
\text { hospital-based group } \\
(-87.9 \mathrm{~mL} \text {; range }-209 \text { to } \\
33.2 \mathrm{~mL} \text { ) and home } \\
\text { monitoring group } \\
\text { (-7.9 } \mathrm{mL} \text {; range }-96 \text { to } \\
69.4 \mathrm{~mL} ; \mathrm{p}=0.25) \\
\text { (3) Correlation between } \\
\text { home and hospital } \\
\text { spirometry was high at all } \\
\text { time-points }(\mathrm{r}=0.97, \\
\mathrm{p}<0.001 \text { at baseline and } \\
12 \text { weeks; } \mathrm{r}=0.96, \mathrm{p}<0.001 \\
\text { at } 24 \text { weeks) } \\
\text { (4) Correlation between } \\
\text { slopes was moderately } \\
\text { strong ( } \mathrm{r}=0.58 ; \mathrm{p}<0.001 \text { ) }\end{array}$ \\
\hline $\begin{array}{l}\text { MAHER et al. } \\
(2020) \text { [38] }\end{array}$ & RCT & $\begin{array}{c}\mathrm{n}=253 \\
\text { Intervention } \\
(\mathrm{n}=127) \\
\text { Age (mean): } \\
70 \text { years (range } \\
61.0-76.0) \\
\text { Male: } 70(55 \%) \\
\text { Placebo ( } \mathrm{n}=126) \\
\text { Age (mean): } \\
69 \text { years (range } \\
63.0-74.0) \\
\text { Male: } 69(55 \%)\end{array}$ & Unclassifiable ILD & $\begin{array}{l}\text { Spirometry } \\
\text { 6MWD, } \\
\text { UCSD-SOBQ, } \\
\text { Leicester Cough } \\
\text { Questionnaire, } \\
\text { SGRQ (baseline } \\
\text { and at } 24 \text { weeks) }\end{array}$ & FVC (once daily) & 24 weeks & $\begin{array}{l}\text { The mean change in } \\
\text { FVC measured by } \\
\text { daily home-based } \\
\text { spirometry, change } \\
\text { in FVC measured by } \\
\text { site spirometry, } \\
\text { change in 6MWD, } \\
\text { change in } \\
\text { UCSD-SOBQ }\end{array}$ & Good & $\begin{array}{l}\text { (1) The primary end-point } \\
\text { was not adequately } \\
\text { analysed due to technical } \\
\text { issues resulting in } \\
\text { variability in home-based } \\
\text { spirometry measurements } \\
\text { (2) Mean FVC decline } \\
\text { measured by clinic } \\
\text { spirometry was less in } \\
\text { pirfenidone than placebo } \\
\text { group (treatment } \\
\text { difference } 95.3 \mathrm{~mL} ; 95 \% \mathrm{Cl} \\
\text { 35.9-154.6, } \mathrm{p}=0.002 \text { ) }\end{array}$ \\
\hline
\end{tabular}


TABLE 3 Continued

\begin{tabular}{|c|c|c|c|c|c|c|c|c|}
\hline Study & $\begin{array}{l}\text { Setting/ } \\
\text { design }\end{array}$ & $\begin{array}{l}\text { Sample size and } \\
\text { characteristics }\end{array}$ & $\begin{array}{l}\text { Disease } \\
\text { group }\end{array}$ & $\begin{array}{l}\text { Clinic measures/ } \\
\text { frequency }\end{array}$ & $\begin{array}{l}\text { Home measures/ } \\
\text { frequency }\end{array}$ & $\begin{array}{l}\text { Study } \\
\text { length }\end{array}$ & Outcome & Quality \\
\hline $\begin{array}{l}\text { RusSELL et al. } \\
\quad(2016) \text { [31] }\end{array}$ & UK/PCS & $\begin{array}{c}\quad n=50 \\
\text { Male: } 45(90 \%) \\
\text { Age (mean } \pm \text { sD): } \\
66.7 \pm 7.9 \text { years }\end{array}$ & IPF & $\begin{array}{c}\text { Spirometry } \\
\text { baseline, and at } 3 \text {, } \\
6 \text { and } 12 \text { months }\end{array}$ & $\begin{array}{l}\mathrm{FEV}_{1}, \mathrm{FVC} \text { (once } \\
\text { daily) }\end{array}$ & $\begin{array}{l}\text { Median: } \\
279 \text { days, } \\
\text { range } 13- \\
490 \text { days }\end{array}$ & $\begin{array}{c}\text { Feasibility and } \\
\text { reliability of } \\
\text { measuring daily FVC }\end{array}$ & Good \\
\hline
\end{tabular}




\begin{tabular}{|c|c|c|c|c|c|c|c|c|c|}
\hline Study & $\begin{array}{l}\text { Setting/ } \\
\text { design }\end{array}$ & $\begin{array}{l}\text { Sample size and } \\
\text { characteristics }\end{array}$ & $\begin{array}{l}\text { Disease } \\
\text { group }\end{array}$ & $\begin{array}{l}\text { Clinic measures/ } \\
\text { frequency }\end{array}$ & $\begin{array}{l}\text { Home measures/ } \\
\text { frequency }\end{array}$ & $\begin{array}{l}\text { Study } \\
\text { length }\end{array}$ & Outcome & Quality & Results \\
\hline $\begin{array}{r}\text { EDWARDs et al. } \\
(2020) \text { [39] }\end{array}$ & $\begin{array}{l}\text { IE/USA/ } \\
\text { PCS }\end{array}$ & $\begin{array}{c}\mathrm{n}=36 \\
\text { USA: Age (mean): } \\
62 \text { years } \\
\text { Ireland: Age } \\
\text { (mean): } 66 \text { years }\end{array}$ & $\mathrm{PF}$ & & $\begin{array}{l}\text { FVC (once daily) } \\
\text { mMRC (once daily) } \\
\text { IPF-PROM (weekly) }\end{array}$ & 1 year & $\begin{array}{l}\text { Acceptability and } \\
\text { utility of } \\
\text { patientMpower }\end{array}$ & Fair & $\begin{array}{l}\text { (1) } 93 \% \text { of respondents } \\
\text { reported a positive impact } \\
\text { on their well-being } \\
\text { (2) Good correlation } \\
\text { between hospital-based } \\
\text { and home-based } \\
\text { spirometry }\end{array}$ \\
\hline $\begin{array}{l}\text { Moor et al. } \\
\text { (2019) [45] }\end{array}$ & NL/PCS & $\begin{array}{c}n=10 \\
\text { Male: } 6(60 \%) \\
\text { Age (mean): } \\
53 \text { years }\end{array}$ & Sarcoidosis & $\begin{array}{c}\text { Spirometry, } \\
\text { activity, PROM } \\
\text { (baseline and at } \\
1 \text { month), patients' } \\
\text { KSQ, EQ-5D-5L, } \\
\text { HADS, FAS } \\
\text { Satisfaction } \\
\text { (interview) }\end{array}$ & $\begin{array}{l}\text { PEF, FEV }{ }_{1}, \mathrm{FVC} \\
\text { (daily) } \\
\text { VAS fatigue, } \\
\text { dyspnoea, cough, } \\
\text { well-being } \\
\text { (weekly) }\end{array}$ & 4 weeks & $\begin{array}{l}\text { Evaluate feasibility } \\
\text { of home monitoring } \\
\text { programme and } \\
\text { patient satisfaction } \\
\text { programme }\end{array}$ & Fair & $\begin{array}{c}\text { (1) Home spirometry } \\
\text { measurements highly } \\
\text { correlated with in-hospital } \\
\text { measurements of FVC } \\
(r=0.97, p<0.001) \text { and } F E V_{1} \\
(r=0.96, p<0.001) \\
\text { (2) Mean adherence to } \\
\text { daily spirometry was } \\
94.6 \% \text {; it was measured } \\
\text { by dividing the total } \\
\text { number of measurements } \\
\text { by the total numbers of } \\
\text { days }\end{array}$ \\
\hline $\begin{array}{l}\text { Moor et al. } \\
\text { (2018) [40] }\end{array}$ & NL/PCS & $\begin{array}{l}n=10 \\
\text { Male: } 9(90 \%) \\
\text { Age (mean): } \\
71 \text { years }\end{array}$ & IPF & $\begin{array}{c}\text { Spirometry, } \\
\text { patient-reported } \\
\text { outcome (baseline } \\
\text { and at } 1 \text { month), } \\
\text { patients' K-BILD, } \\
\text { HADS, EuroQoL } \\
\text { 5D-5 L }\end{array}$ & $\begin{array}{l}\text { Home spirometry } \\
\text { (daily) } \\
\text { Patient-reported } \\
\text { outcome (weekly) }\end{array}$ & 4 weeks & $\begin{array}{l}\text { Feasibility of a } \\
\text { pre-developed } \\
\text { home monitoring } \\
\text { programme in IPF } \\
\text { (home spirometry) }\end{array}$ & Fair & $\begin{array}{l}\text { (1) Home-based } \\
\text { spirometry showed similar } \\
\text { results to hospital-based } \\
\text { spirometry; measurements } \\
\text { of home and hospital FVC } \\
\text { were correlated ( } r=0.94 ; \\
p<0.001 \text { ) and FEV }(r=0.97 \text {; } \\
\text { p<0.001) were highly } \\
\text { correlated } \\
\text { (2) Feasibility and } \\
\text { potential barriers of home } \\
\text { spirometry: } 80 \% \text { of } \\
\text { patients reported easy to } \\
\text { use and } 90 \% \text { said it was } \\
\text { not burdensome; mean } \\
\text { adherence was } 98.8 \% \text { to } \\
\text { home monitoring } \\
\text { programme }\end{array}$ \\
\hline
\end{tabular}

Continued 


\begin{tabular}{|c|c|c|c|c|c|c|c|c|c|}
\hline Study & $\begin{array}{l}\text { Setting/ } \\
\text { design }\end{array}$ & $\begin{array}{l}\text { Sample size and } \\
\text { characteristics }\end{array}$ & $\begin{array}{l}\text { Disease } \\
\text { group }\end{array}$ & $\begin{array}{l}\text { Clinic measures/ } \\
\text { frequency }\end{array}$ & $\begin{array}{l}\text { Home measures/ } \\
\text { frequency }\end{array}$ & $\begin{array}{l}\text { Study } \\
\text { length }\end{array}$ & Outcome & Quality & Results \\
\hline $\begin{array}{l}\text { Moor et al. } \\
\qquad(2020)[46]\end{array}$ & NL/PCS & $\begin{array}{c}\mathrm{n}=50 \\
\text { ( } \mathrm{n}=44 \text { acceptable } \\
\text { data) } \\
\text { Age range: } 43-79 \\
\text { years } \\
\text { Male: } 68 \%\end{array}$ & IPF & $\begin{array}{l}\text { Questionnaire } \\
\text { (baseline and at } \\
6 \text { weeks) }\end{array}$ & $\begin{array}{l}\text { FVC (twice daily) } \\
\text { Patient-reported } \\
\text { K-BILD online }\end{array}$ & 6 weeks & $\begin{array}{l}\text { Measure diurnal } \\
\text { variation in FVC in } \\
\text { patients with f-ILD } \\
\text { using home } \\
\text { spirometry, evaluate } \\
\text { the relationship } \\
\text { between FVC and } \\
\text { activity, } \\
\text { home-based FVC, } \\
\text { home and } \\
\text { hospital-based } \\
\text { correlation }\end{array}$ & Fair & $\begin{array}{l}\text { (1) Morning FVC was } \\
\text { significantly higher than } \\
\text { afternoon FVC (mean } \\
\text { difference } 36 \mathrm{~mL}, \\
\text { p }<0.001) \text {; the mean } \\
\text { difference between } \\
\text { morning and afternoon } \\
\text { FVC was similar for } \\
\text { patients with IPF } \\
\text { compared with all f-ILDs } \\
\text { (2) Daily step correlated } \\
\text { with FVC }(r=0.32, p=0.028 \text {, } \\
\text { K-BILD total score ( } r=0.5 \text {, } \\
p<0.001) \text { ) } \\
\text { (3) Home and } \\
\text { hospital-based spirometry } \\
\text { were correlated ( } r=0.98, \\
p<0.0001)\end{array}$ \\
\hline $\begin{array}{l}\text { Broos et al. } \\
\text { (2017) [41] }\end{array}$ & NL/PCS & $\begin{array}{c}\mathrm{n}=21 \\
\text { Male: } 13 \\
\text { Female: } 8 \\
\text { Age (mean } \pm \text { sD): } \\
43 \pm 11 \text { years } \\
76 \% \text { diagnosed } \\
\text { with Scadding } \\
\text { stage II sarcoidosis }\end{array}$ & Sarcoidosis & $\begin{array}{l}\text { Clinic spirometry, } \\
\text { SGRQ, SF-36, KSQ, } \\
\text { MRC, FAS at } \\
\text { baseline, } 1 \text { and } \\
3 \text { months }\end{array}$ & $\begin{array}{c}\text { FVC (daily) } \\
\text { MRC, FAS (weekly) }\end{array}$ & 3 months & $\begin{array}{l}\text { Detect early steroid } \\
\text { treatment effects in } \\
\text { newly treated } \\
\text { pulmonary } \\
\text { sarcoidosis }\end{array}$ & Good & $\begin{array}{l}\text { (1) Home spirometry in } \\
\text { sarcoidosis is reliable } \\
\text { (2) Home and hospital } \\
\text { spirometry were } \\
\text { correlated }(r=0.98 \text {; } \\
p<0.001)\end{array}$ \\
\hline $\begin{array}{l}\text { Marcoux et al. } \\
\text { (2019) [28] }\end{array}$ & NL/PCS & $\begin{array}{c}\quad n=20 \\
\text { Male: } 16(80 \%) \\
\text { Age (mean } \pm \text { sD): } \\
73 \pm 6.9 \text { years }\end{array}$ & IPF & $\begin{array}{c}\text { Clinic spirometry at } \\
\text { baseline, } 4 \text { and } 23 \\
\text { weeks } \\
\text { 6MWD (baseline } \\
\text { and at } 12 \text { weeks) }\end{array}$ & $\begin{array}{c}\text { FVC (3 } \\
\text { manoeuvres daily) }\end{array}$ & 12 weeks & $\begin{array}{l}\text { Test the } 12 \text {-week } \\
\text { feasibility of blinded } \\
\text { daily handheld } \\
\text { spirometry and } \\
\text { physical activity } \\
\text { monitoring in } \\
\text { patients with IPF }\end{array}$ & Good & $\begin{array}{l}\text { (1) The correlation for } \\
\text { office-based and handheld } \\
\text { FVC measurements was } \\
0.99(95 \% \mathrm{Cl} 0.97-0.99) \\
\text { and } 0.95 \text { ( } 95 \% \mathrm{Cl} 0.91- \\
0.98) \text {, respectively } \\
\text { (2) Mean adherence to } \\
\text { home spirometry was } 84 \%\end{array}$ \\
\hline
\end{tabular}




\begin{tabular}{|c|c|c|c|c|c|c|c|c|c|}
\hline Study & $\begin{array}{l}\text { Setting/ } \\
\text { design }\end{array}$ & $\begin{array}{l}\text { Sample size and } \\
\text { characteristics }\end{array}$ & $\begin{array}{l}\text { Disease } \\
\text { group }\end{array}$ & $\begin{array}{l}\text { Clinic measures/ } \\
\text { frequency }\end{array}$ & $\begin{array}{l}\text { Home measures/ } \\
\text { frequency }\end{array}$ & $\begin{array}{l}\text { Study } \\
\text { length }\end{array}$ & Outcome & Quality & Results \\
\hline $\begin{array}{l}\text { Noth et al. } \\
\quad(2021) \text { [43] }\end{array}$ & NL/PCS & $\begin{array}{l}\mathrm{n}=346 \text { diagnosed } \\
\text { with IPF in the } \\
\text { previous } 3 \text { years } \\
\text { and had an FVC } \\
\geqslant 80 \% \text { predicted; } \\
116 \text { randomised to } \\
\text { nintedanib, } 230 \\
\text { randomised to } \\
\text { placebo for } \\
12 \text { weeks, followed } \\
\text { by an open-label } \\
\text { period in which all } \\
\text { subjects received } \\
\text { nintedanib } 150 \mathrm{mg} \\
\text { twice daily for } 40 \\
\text { weeks }\end{array}$ & IPF & $\begin{array}{c}\text { Clinic spirometry at } \\
\text { baseline and weeks } \\
\begin{array}{c}4,8,12,16,20,24 \\
36 \text { and } 52\end{array}\end{array}$ & FVC (weekly) & 1 year & $\begin{array}{l}\text { Investigate the } \\
\text { feasibility and } \\
\text { validity of home } \\
\text { spirometry as a } \\
\text { measure of lung } \\
\text { function decline in } \\
\text { patients with IPF }\end{array}$ & Good & $\begin{array}{l}\text { (1) Over } 52 \text { weeks, mean } \\
\text { adherence was } 86 \% \\
\text { (2) Strong correlations } \\
\text { were observed between } \\
\text { home- and } \\
\text { clinic-measured FVC at all } \\
\text { time-points ( } r=0.72 \text { to } \\
\text { 0.84), but correlations } \\
\text { between home- and } \\
\text { clinic-measured rates of } \\
\text { change in FVC were weak } \\
\text { (r=0.26 for rate of decline } \\
\text { in FVC over } 52 \text { weeks); the } \\
\text { correlations were weaker } \\
\text { in subjects who provided } \\
\text { more FVC readings per } \\
\text { week, due to variability in } \\
\text { change in FVC measured } \\
\text { at home (greater number } \\
\text { of outliers) and errors in } \\
\text { measurements }\end{array}$ \\
\hline $\begin{array}{l}\text { Moor et al. } \\
\quad(2020)[42]\end{array}$ & NE/PCS & $\begin{array}{c}\mathrm{n}=10 \\
\text { Female: } 60 \% \\
\text { Age (mean } \pm \mathrm{sD}): \\
60.3 \pm 9.9 \text { years }\end{array}$ & $\begin{array}{c}\text { Systemic } \\
\text { sclerosis-associated } \\
\text { ILD }\end{array}$ & $\begin{array}{l}\text { Spirometry at } \\
\text { baseline and } \\
3 \text { months } \\
\text { K-BILD, HADS, } \\
\text { EQ-5D-5L (baseline } \\
\text { and at } 6 \text { weeks) }\end{array}$ & FVC (once daily) & 3 months & $\begin{array}{l}\text { Investigate the } \\
\text { feasibility of an } \\
\text { online home } \\
\text { monitoring } \\
\text { application, and } \\
\text { spirometry }\end{array}$ & Good & $\begin{array}{l}\text { (1) Mean } \pm \text { SD adherence } \\
\text { was } 98.8 \pm 1.5 \% \\
\text { (2) Strong adherence and } \\
\text { acceptability; } 90 \% \text { found } \\
\text { home monitoring pleasant } \\
\text { and wanted to continue } \\
\text { to use home monitoring } \\
\text { application daily }\end{array}$ \\
\hline $\begin{array}{l}\text { IPF: idiopathic } \\
\text { Rating Change } \\
\text { Interstitial Lun } \\
\text { St George's R } \\
\text { IPF-PROM: Idic } \\
\text { SF36: 36-Item }\end{array}$ & $\begin{array}{l}\text { onary fi } \\
\text { OL: hea } \\
\text { ase; NL } \\
\text { ory Qu } \\
\text { E Pulme } \\
\text { Form; U }\end{array}$ & $\begin{array}{l}\text { sis; CAT: COPD As } \\
\text { elated quality of } \\
\text { etherlands; PESal } \\
\text { nnaire; UK: Unite } \\
\text { y Fibrosis (IPF) P } \\
\text {-SOBQ: The Univ }\end{array}$ & $\begin{array}{l}\text { nent Test; EQ-5D-5L } \\
\text { ILD: interstitial lung } \\
\text { tient Experiences a } \\
\text { ngdom; US: United } \\
\text { tt Reported Outcom } \\
\text { / of California, San D }\end{array}$ & $\begin{array}{l}\text { uroQoL Five-Dime } \\
\text { sease; 6MWD: 6-m } \\
\text { Satisfaction with } \\
\text { ates; VAS: visual a } \\
\text { Measure; KSQ: Kir } \\
\text { so, Shortness of B }\end{array}$ & $\begin{array}{l}\text { Is Questionnair } \\
\text { lk distance; HA } \\
\text { cation question } \\
\text { ue scale; mMR } \\
\text { Sarcoidosis Que } \\
\text { Questionnaire. }\end{array}$ & $\begin{array}{l}\text { 1: forced } \\
\text { ospital A } \\
\text { PCS: pr } \\
\text { dified Me } \\
\text { naire; PE }\end{array}$ & $\begin{array}{l}\text { atory volume in } \\
\text { and Depressior } \\
\text { tive cohort stud } \\
\text { Research Counc } \\
\text { ak expiratory flo }\end{array}$ & $\begin{array}{l}\text { VC: fo } \\
\text {; IE: I } \\
\text { T: ranc } \\
\text {-VAS: } \\
\text {-D: fib }\end{array}$ & $\begin{array}{l}\text { vital capacity; GRC: Global } \\
\text { d; K-BILD: The King’s Brief } \\
\text { sed controlled trial; SGRQ: } \\
\text { Qol-visual analogue scales; } \\
\text { g interstitial lung disease; }\end{array}$ \\
\hline
\end{tabular}


from 10 to 346 patients, with ages ranging from 31 to 73 years. The majority of the patients were male. The RCT duration was 24 weeks, and the prospective cohort studies ranged from 2 to 70 weeks.

A variety of types of home monitoring techniques, including but not limited to spirometry, weekly symptom reporting through electronic means, wearable devices to track/monitor vitals and activity, were used. Table 4 summarises the techniques and tools used in the included studies.

\section{Feasibility \\ Feasibility}

A recent study by MAHER et al. [38] raised some concerns related to the data integrity of home-based spirometry in patients with pulmonary fibrosis due to high variability and technical problems. However, 13 studies support the feasibility and utility of home-based spirometry in patients with ILD [20, 28, 30-32, 38-43, 45, 46]. Moor et al. [30] reported that the slopes of home- and clinic-based FVC over time were comparable. The rate of discontinuation was not dissimilar to the rates seen in other clinical trials. Most participants were able to provide daily readings, with at least four every 5 days, for up to 1 year [30, 31, 39, 42]. In contrast, VeIt et al. [32] and Marcoux et al. [28] required patients to perform three spirometry manoeuvres every day and found it feasible. In summary, regular home measurement of FVC in the context of a clinical study was found to be feasible. JohAnNson et al. [20] and Notн et al. [43] showed feasibility and reliability of weekly spirometry in patients with ILD. Two studies in sarcoidosis [41, 45] and one in IPF [29] included 121 patients who performed daily home spirometry, and completed patient-reported outcomes at baseline, weekly and at the end of the studies. The articles concluded that a home monitoring programme for IPF and sarcoidosis was indeed feasible, and was well tolerated by most of the patients [29, 30, 41].

In general, all the included studies presented a positive experience in relation to acceptance of home monitoring programmes by patients. EDWARDs et al. [39] demonstrated acceptability of home monitoring to patients at 6 weeks, and the majority of patients wished to continue with home monitoring beyond this time-point. Patients showed a positive attitude towards home monitoring techniques despite differences in age and the size of studies. Ease of use and friendliness of technology contributed to good compliance among patients and their acceptance of the home monitoring systems [34].

\section{Adherence}

Adherence was calculated by the number of home measurements divided by the number of weeks enrolled in the study for weekly measurements, and number of home measurements divided by the number of days enrolled in the study for daily measurements [20, 32]. In the study by JoHANnson et al. [20] mean adherence to three times weekly home FVC monitoring over 24 weeks was $90.5 \%$ (sD=18.3\%). The VEIT et al. [32] study reported that adherence to three times daily home spirometry decreased over time. Median adherence dropped within the first 28 days and decreased from $90 \%$ to $81 \%$ over 6 months. Acceptance, however, was high; only four patients discontinued within the first week as dyspnoea made it too difficult

TABLE 4 The components and outcomes measured in the home monitoring intervention in this systematic review

\begin{tabular}{|c|c|c|c|c|c|c|c|c|c|}
\hline \multirow[t]{2}{*}{ Study } & \multicolumn{5}{|c|}{ Measurement parameters } & \multicolumn{3}{|c|}{ Data transmission } & \multirow{2}{*}{$\begin{array}{c}\text { Online } \\
\text { platform/app }\end{array}$} \\
\hline & Spirometer & $\begin{array}{l}\text { Quality } \\
\text { check }\end{array}$ & Oximetry & $\begin{array}{l}\text { Step count/ } \\
\text { 6MWD }\end{array}$ & $\begin{array}{l}\text { Symptom } \\
\text { report }\end{array}$ & $\begin{array}{c}\text { Downloaded by } \\
\text { staff }\end{array}$ & Real-time & $\begin{array}{l}\text { Diary } \\
\text { card }\end{array}$ & \\
\hline Moor et al. (2020) [30] & $\checkmark$ & $\checkmark$ & & & $\checkmark$ & & $\checkmark$ & & $\checkmark$ \\
\hline Russell et al. (2016) [31] & $\checkmark$ & & & & & & & $\checkmark$ & \\
\hline Johannson et al. (2017) [20] & $\checkmark$ & $\checkmark$ & & & $\checkmark$ & $\checkmark$ & & & \\
\hline Moor et al. (2019) [45] & $\checkmark$ & $\checkmark$ & & $\checkmark$ & $\checkmark$ & & $\checkmark$ & & $\checkmark$ \\
\hline VeIt et al. (2020) [32] & $\checkmark$ & $\checkmark$ & & $\checkmark$ & $\checkmark$ & $\checkmark$ & & & \\
\hline Moor et al. (2018) [40] & $\checkmark$ & $\checkmark$ & & & $\checkmark$ & & $\checkmark$ & & $\checkmark$ \\
\hline EdwARDs et al. (2020) [39] & $\checkmark$ & $\checkmark$ & $\checkmark$ & $\checkmark$ & $\checkmark$ & & $\checkmark$ & & $\checkmark$ \\
\hline Nотн et al. (2020) [43] & $\checkmark$ & $\checkmark$ & & & & & & & \\
\hline Moor et al. (2020) [42] & $\checkmark$ & $\checkmark$ & & $\checkmark$ & $\checkmark$ & & $\checkmark$ & & $\checkmark$ \\
\hline Broos et al. (2017) [41] & $\checkmark$ & & & & $\checkmark$ & $\checkmark$ & & & \\
\hline Marcoux et al. (2019) [28] & $\checkmark$ & $\checkmark$ & & $\checkmark$ & & & $\checkmark$ & & $\checkmark$ \\
\hline Moor et al. (2020) [46] & $\checkmark$ & & & $\checkmark$ & & & $\checkmark$ & & $\checkmark$ \\
\hline MAHER et al. (2020) [38] & $\checkmark$ & $\checkmark$ & & $\checkmark$ & $\checkmark$ & $\checkmark$ & & & \\
\hline
\end{tabular}


to perform daily measurements. Nотн et al. [43] demonstrated that adherence to weekly home spirometry decreased with time but remained over $75 \%$ throughout the entirety of the study. Studies by Moor et al. [29, 30, 40, 42, 45] demonstrated that mean adherence to once daily home FVC ranged from $90.5 \%$ to $98.8 \%$. In summary, adherence to a home monitoring programme varied depending on study duration, frequency of measurement and transmission. However, nine studies [20, 28, 30, 32, 39, 40, 42, 43, 45] reported that mean adherence to home spirometry was $>75 \%$. Vert et al. [32] reported that the decrease in adherence with time might be due to lack of reminders to perform spirometry. Moor et al. [30] found good adherence that did not decrease with time and suggested that in other studies such challenges with home spirometry might have arisen because patients were blinded from their results and had no technical helpdesk.

Utility

Prediction of disease progression and mortality

Only Russell et al. [31] and Veit et al. [32] reported information describing whether home-based monitoring was able to detect disease progression. Russell et al. [31] reported 18 deaths out of 50 subjects during the 490-day study of patient-recorded daily spirometry. The study compared the rate of change in FVC between baseline to 28 days, 3 months, 6 months and 12 months. It demonstrated that the rate of change in FVC was more predictive for disease progression and mortality when measured at 3 months

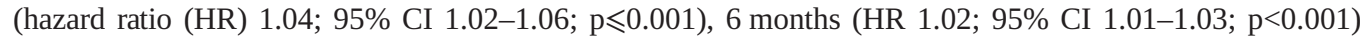
and 12 months (HR 1.012; 95\% CI 1.007-1.01; p=0.001); 28 days did not yield a significant correlation [31].

VEIT et al. [32] included 47 patients and provided reliable daily measurements of spirometry for the cohort study. The study defined disease progression as death due to respiratory failure, lung transplantation, AE or hospital-based relative FVC decline $>10 \%$ at 3 or 6 months [32]. During the 6 months, 12 of these 40 participants experienced disease progression [32]. A group of patients displayed high daily variability in FVC during the initial 28 days of the study; $60.0 \%$ showed a variation $\geqslant 5 \%$. FVC variability over 28 days was independently associated with disease progression (HR 1.20; 95\% CI 1.05-1.3; p=0.007). FVC variability over 3 months was also a significant predictor for disease progression (HR 1.2; 95\% CI 1.01-1.64; $\mathrm{p}=0.03$ ). It is possible that individual techniques for performing daily spirometry could cause the variation seen in FVC results early on. This study examined results prior to and post the 3-month hospital FVC check and saw no significant variations. This helps eliminate individual technique as a contributor to varied results, and so the correlation between results of 28 days and 3 months being strong, significantly supports the relationship between change in FVC and disease progression. The 6-min walk distance (6MWD) ( $301 \pm 140 \mathrm{~m}$ versus $433 \pm 89 \mathrm{~m} ; \mathrm{p}=0.009)$ and the King's Brief Interstitial Lung Disease questionnaire (K-BILD) total score $(46.3 \pm 8.1$ versus $55.8 \pm 12.7$; $\mathrm{p}=0.004)$ were lower in the progressive group, indicating more limitation of physical and subjective well-being [34].

\section{Correlation between home-based spirometry and hospital-based monitoring}

Nine studies [28, 30-32, 39, 41, 42, 45, 46] confirmed strong correlation between home and hospital-based spirometry readings of FVC. Six studies [30, 36, 41, 42, 45, 46] showed a significant positive correlation with a p-value $<0.001$ and $r=0.93-0.98$. Nотн et al. [43] reported strong correlations between home- and clinic-measured FVC at all time-points ( $\mathrm{r}=0.72$ to 0.84 ), but correlations between home- and clinic-measured rates of change in FVC were weak ( $\mathrm{r}=0.26$ for rate of decline in FVC over 52 weeks). The correlations were weaker in subjects who provided more FVC readings per week. This was due to variability in change in FVC measured at home (greater number of outliers) and errors in measurements [43]. Variability in change from baseline in FVC was greater when measured by home rather than clinic spirometry [43]. However, JoHANnson et al. [20] and MAHER et al. [50] report studies that had problems with measurement variability, and the quality assurance of home-based spirometry is a major consideration. JoHANnson et al. [20] showed that home-based monitoring of FVC value was variable and hence suggested hospital-based confirmation of FVC decline to prevent error, defeating the object of home monitoring. Similarly, MAHER et al. [50] recommended further research before home measurements of FVC be used as a primary end-point in clinical trials and in particular, a need for a priori consideration of how the planned statistical analysis will handle data from patients with missing or variable spirometry values, so as not to affect planned statistical analyses [40,43]. Variability might also have been caused by limited adherence, training, technical problems and lack of reminders [19, 29, 40]. MARcoux et al. [28] reported that correlation between home spirometer and office-based measurements declined at week 12.

Home versus hospital monitoring of medication use

Two studies by Moor et al. [30, 45] used an online eHealth application developed for patients with sarcoidosis and IPF. Patients kept track of their own health-related data, such as lung function, symptoms, medication and side-effects, and were provided with a graphical overview of their data. It was found that 
patients reported better insights into the effects of medication by seeing a daily overview of their lung function and potential disease progression. This suggests that patients with ILD had better-tailored treatment decisions during home monitoring programmes [30, 45]. Moreover, Broos et al. [41] suggest that home monitoring of physiological parameters could help physicians not only to detect disease progression but also to evaluate response to therapy.

Home versus hospital monitoring of well-being and health-related quality of life

Moor et al. [30] assessed health-related quality of life in patients with IPF using the K-BILD questionnaire in a 24-week randomised controlled trial. K-BILD is a validated, 15-item self-rated questionnaire, and an interstitial lung disease-specific, health-related quality of life questionnaire [30]. Home monitoring was not associated with a statistically significant improvement in K-BILD (mean difference 2.67 points; 95\% CI $-1.85-7.17$; $\mathrm{p}=0.24$ ). The RCT consisted of 90 patients; 46 out of 90 received home monitoring services. It was found that both the mean K-BILD score and the K-BILD psychological domain score was greater in the home monitoring group indicating improved general psychological well-being. The results of the RCT showed that the anxiety scores were low in both groups, improved psychological well-being and allowed for individually tailored treatment adjustments.

\section{Discussion}

We have conducted the first systematic review examining home monitoring of lung function and symptoms to detect ILD exacerbations and progression. 13 studies utilised home monitoring to measure feasibility and utility with two studies including disease progression as an outcome. The included studies provide evidence to support the feasibility and utility of home-based monitoring in patients with ILD [20, 28, 30 32, 38-43, 45, 46]. However, the included studies varied in their primary outcome, and were heterogeneous with respect to duration, measurements, and the type of technology and questionnaires used.

ILD relies on regular pulmonary function testing to guide management [6, 47-50]. Among the tools used for home-based monitoring, comparison of spirometry before and after an exacerbation and/or progression demonstrated that FVC decline reflects the severity of disease progression [2, 42, 51]. At present, spirometry is the primary test used to detect exacerbation and/or progression in ILD [52-54]. FVC variability was addressed in two of the included studies in patients with ILD. Veit et al. [32] and RusseLL et al. [31] demonstrated a link between variability of FVC and ILD disease progression [31, 32]. VEIT et al. [32] found that FVC variability was a statistically significant predictor for disease progression [53, 55, 56]. Moreover, Russell et al. [31] confirmed that the rate of change in FVC was most predictive for disease progression and mortality when measured over 3, 6 and 12 months. Risk of variation can be mitigated by providing training, a technical helpdesk and real-time monitoring with reminders [30].

Early detection of disease progression and AE has been a focus for several diseases, especially in COPD [57]. Home monitoring of heart rate and oxygen saturation has been shown to result in early detection of exacerbation in COPD [26]. Similarly, previously studies reported exacerbation and/or progression in patients with ILD [6, 21, 22, 23]. Most studies until recently have only focused on the feasibility and reliability of home monitoring. However, the potential for home monitoring to facilitate early identification of AE-ILD has gained increasing interest during the past years. Recently, Moor et al. [40] reported that FVC decline could be detected 2 days before symptoms of infection began. Although it is possible to continuously monitor heart rate and oxygen saturation, the included studies in this review did not examine data for heart rate and oxygen saturation. Monitoring heart rate, 6MWD, activity and oxygen saturation remotely might contribute to a more precise prediction of disease exacerbation and/or progression [50, 53, 58-65].

Other factors to be considered are potential use of real-time monitoring and artificial intelligence to predict exacerbation and/or progression [60, 66-68]. Artificial intelligence is now adapted to interpret complex data in COPD to predict acute respiratory events [60, 69-73]. Artificial intelligence could allow monitoring of a large number of patients continuously and simultaneously. Utilising machine learning via an online platform with real-time data transmission could allow real-time detection of exacerbation and/or progression [53, 58, 74]. Predictors of disease exacerbation and/or progression have already been published but not specifically for patients with ILD [69, 75-77]. The ability to accurately detect rare occurrences, such as AE-IPF, offers the potential for clinical trials to assess early treatments for these, often devastating, events. We believe that machine learning and the use of an online platform with direct data transmission, of proven value in other diseases [69, 72], has promising potential in the field of ILD.

Encouraging findings were the high level of adherence and that patients were generally satisfied with home monitoring [29, 30, 39, 40]. Nevertheless, some studies showed that adherence to home-based monitoring decreased over time [20, 32, 78]. It is suggested that involving patients in monitoring their own condition 
would give some feeling of being in control and managing the condition [20, 79, 80]. This aligns with what has been suggested in respect to patients' increase in adherence to, and satisfaction with, home-based monitoring [20, 39, 81, 82]. Another reason for patients to remain adherent might be the ability of home monitoring to generate early alerts of deterioration with the potential for early interventions [30].

In recent assessments of home monitoring for patients with ILD, healthcare providers were interested to use home monitoring for regular care and research purposes [83]. Although these findings are encouraging, further studies considering clinician perspectives are necessary. It is important to consider that the total number of participants was fewer than one thousand, and all were patients. This is not a complete representation of real-world clinical experience. Both patients and clinicians could benefit from effective home monitoring for patients with ILD to detect exacerbations and/or progression and to allow timelier intervention; for closer monitoring of therapeutic interventions; and to assess novel medications in clinical trials. The potential cost reduction on both patient and clinician sides could also be explored.

The potential of home monitoring of ILD patients was a consistent focus of the included studies, but the challenge of providing effective detection of exacerbation and/or progression has yet to be addressed. The published trials were mostly feasibility studies with a few ongoing studies [56, 84-86] focusing on detection of AE-ILD using home spirometry. Researchers should conduct longitudinal studies of physiological parameters and symptoms with real-time feedback from integrated spirometry, pulse oximetry and wearable devices and smartphone applications to assess their ability to detect exacerbation and/or progression in patients with ILD.

\section{Conclusion}

Although there were no studies reporting conclusively on the ability of home monitoring to detect deteriorations of ILD and AE-ILD, this systematic review suggests good adherence and feasibility of home monitoring. Home monitoring presents an opportunity for earlier detection of exacerbation and/or progression in ILD and examining this question should be the focus of future research.

Provenance: Submitted article, peer reviewed.

Conflict of interest: M.A. Althobiani has nothing to disclose. R.A. Evans has nothing to disclose. J.S. Alqahtani has nothing to disclose. A.M. Aldhahir has nothing to disclose. A-M. Russell reports speaker fees, conference support, research support and consultancy fees from Boehringer Ingelheim, and speaker fees from Hoffman La Roche, outside the submitted work. A-M. Russell is a NIHR70@70 Senior Research Fellow. The views expressed in this publication are those of the author(s) and not necessarily those of the National Institute for Health Research or the Dept of Health and Social Care. J.R. Hurst has nothing to disclose. J.C. Porter has nothing to disclose.

Support statement: This study was supported by Dept of Respiratory Therapy, Faculty of Medical Rehabilitation Sciences, King Abdulaziz University, Jeddah, Saudi Arabia through the Saudi Arabian Cultural Bureau in London.

\section{References}

1 Raghu G, Collard HR, Egan JJ, et al. An official ATS/ERS/JRS/ALAT statement: idiopathic pulmonary fibrosis: evidence-based guidelines for diagnosis and management. Am J Respir Crit Care Med 2011; 183: 788-824.

2 Seibold MA, Wise AL, Speer MC, et al. A common MUC5B promoter polymorphism and pulmonary fibrosis. N Engl J Med 2011; 364: 1503-1512.

3 Kropski JA, Pritchett JM, Zoz DF, et al. Extensive phenotyping of individuals at risk for familial interstitial pneumonia reveals clues to the pathogenesis of interstitial lung disease. Am J Respir Crit Care Med 2015; 191: 417-426.

4 Marshall RP, Puddicombe A, Cookson WO, et al. Adult familial cryptogenic fibrosing alveolitis in the United Kingdom. Thorax 2000; 55: 143-146.

5 American Thoracic Society/European Respiratory Society. American Thoracic Society/European Respiratory Society International Multidisciplinary Consensus Classification of the Idiopathic Interstitial Pneumonias. This joint statement of the American Thoracic Society (ATS), and the European Respiratory Society (ERS) was adopted by the ATS board of directors, June 2001 and by the ERS Executive Committee, June 2001. Am J Respir Crit Care Med 2002; 165: 277-304.

6 Ley B, Collard HR, Talmadge E, et al. Clinical course and prediction of survival in idiopathic pulmonary fibrosis. Am J Respir Crit Care Med 2011; 183: 431-440.

7 British Lung Foundation. Lung disease in the UK - big picture statistics. https://statistics.blf.org.uk/ Date last accessed: 11 November 2020.

$8 \quad$ Lederer DJ, Martinez FJ. Idiopathic pulmonary fibrosis. N Engl J Med 2018; 378: 1811-1823. 
9 Lee SH, Kim SY, Kim DS, et al. Predicting survival of patients with idiopathic pulmonary fibrosis using GAP score: a nationwide cohort study. Respir Res 2016; 17: 131.

10 Noble PW, Albera C, Bradford WZ, et al. Pirfenidone in patients with idiopathic pulmonary fibrosis (CAPACITY): two randomised trials. Lancet 2011; 377: 1760-1769.

11 King TE, Bradford WZ, Castro-Bernardini S, et al. A Phase 3 trial of pirfenidone in patients with idiopathic pulmonary fibrosis. N Engl J Med 2014; 370: 2083-2092.

12 Richeldi L, du Bois RM, Raghu G, et al. Efficacy and safety of nintedanib in idiopathic pulmonary fibrosis. N Engl J Med 2014; 370: 2071-2082.

13 Maher TM, Strek ME. Antifibrotic therapy for idiopathic pulmonary fibrosis: time to treat. Respir Res 2019; 20 : 205.

14 Raghu G, Chen SY, Yeh WS, et al. Idiopathic pulmonary fibrosis in US Medicare beneficiaries aged 65 years and older: incidence, prevalence, and survival, 2001-11. Lancet Respir Med 2014; 2: 566-572.

15 Win T, Screaton NJ, Porter JC, et al. Pulmonary (18)F-FDG uptake helps refine current risk stratification in idiopathic pulmonary fibrosis (IPF). Eur J Nucl Med Mol Imaging 2018; 45: 806-815.

16 Karimi-Shah BA, Chowdhury BA. Forced vital capacity in idiopathic pulmonary fibrosis: FDA review of pirfenidone and nintedanib. N Engl J Med 2015; 372: 1189-1191.

17 Spagnolo P, Maher TM. Clinical trial research in focus: why do so many clinical trials fail in IPF? Lancet Respir Med 2017; 5: 372-374.

18 Gross TJ, Hunninghake GW. Idiopathic pulmonary fibrosis. N Engl J Med 2001; 345: 517-525.

19 Wells AU, Behr J, Costabel U, et al. Hot of the breath: mortality as a primary end-point in IPF treatment trials: the best is the enemy of the good. Thorax 2012; 67: 938-940.

20 Johannson KA, Vittinghoff E, Morisset J, et al. Home monitoring improves endpoint efficiency in idiopathic pulmonary fibrosis. Eur Respir J 2017; 50: 1602406.

21 Collard HR, Ryerson CJ, Corte TJ, et al. Acute exacerbation of idiopathic pulmonary fibrosis. An international working group report. Am J Respir Crit Care Med 2016; 194: 265-275.

22 Song JW, Hong SB, Lim CM, et al. Acute exacerbation of idiopathic pulmonary fibrosis: incidence, risk factors and outcome. Eur Respir J 2011; 37: 356-363.

23 Leuschner G, Behr J. Acute exacerbation in interstitial lung disease. Front Med (Lausanne) 2017; 4: 176.

24 Hurst JR, Donaldson GC, Quint JK, et al. Domiciliary pulse-oximetry at exacerbation of chronic obstructive pulmonary disease: prospective pilot study. BMC Pulm Med 2010; 10: 52.

25 Wilkinson TMA, Donaldson GC, Hurst JR, et al. Early therapy improves outcomes of exacerbations of chronic obstructive pulmonary disease. Am J Respir Crit Care Med 2004; 169: 1298-1303.

26 Al Rajeh AM, Aldabayan YS, Aldhahir A, et al. Once daily versus overnight and symptom versus physiological monitoring to detect exacerbations of chronic obstructive pulmonary disease: pilot randomized controlled trial. JMIR Mhealth Uhealth 2020; 8: e17597.

27 Denis F, Basch E, Septans A-L, et al. Two-year survival comparing web-based symptom monitoring vs routine surveillance following treatment for lung cancer. JAMA 2019; 321: 306-307.

28 Marcoux V, Wang M, Burgoyne SJ, et al. Mobile health monitoring in patients with idiopathic pulmonary fibrosis. Ann Am Thorac Soc 2019; 16: 1327-1329.

29 Moor CC, van Manen MJG, Tak NC, et al. Development and feasibility of an eHealth tool for idiopathic pulmonary fibrosis. Eur Respir J 2018; 51: 1702508.

30 Moor CC, Mostard RLM, Grutters JC, et al. Home monitoring in patients with idiopathic pulmonary fibrosis. A randomized controlled trial. Am J Respir Crit Care Med 2020; 202: 393-401.

31 Russell AM, Adamali H, Molyneaux PL, et al. Daily home spirometry: an effective tool for detecting progression in idiopathic pulmonary fibrosis. Am J Respir Crit Care Med 2016; 194: 989-997.

32 Veit T, Barnikel M, Crispin A, et al. Variability of forced vital capacity in progressive interstitial lung disease: a prospective observational study. Respir Res 2020; 21: 270.

33 British Lung Foundation. Coronavirus and COVID-19. Coronavirus and living with a lung condition. https:// www.blf.org.uk/support-for-you/coronavirus/people-living-with-lung-condition Date last accessed: 24 March 2021.

34 Nakshbandi G, Moor CC, Wijsenbeek MS. Home monitoring for patients with ILD and the COVID-19 pandemic. Lancet Respir Med 2020; 8: 1172-1174.

35 Antoniou KM, Raghu G, Tzilas V, et al. Management of patients with interstitial lung disease in the midst of the COVID-19 pandemic. Respiration 2020; 99: 625-627.

36 Moher D, Liberati A, Tetzlaff J, et al. Preferred reporting items for systematic reviews and meta-analyses: the PRISMA statement. BMJ 2009; 339: b2535.

37 Page MJ, McKenzie JE, Bossuyt PM, et al. The PRISMA 2020 statement: An updated guideline for reporting systematic reviews. PLoS Med 2021; 18: e1003583.

38 Maher TM, Corte TJ, Fischer A, et al. Pirfenidone in patients with unclassifiable progressive fibrosing interstitial lung disease: a double-blind, randomised, placebo-controlled, phase 2 trial. Lancet Respir Med 2020; 8: 147-157. 
39 Edwards C, Costello E, Cassidy N, et al. Use of the patientMpower app with home-based spirometry to monitor the symptoms and impact of fibrotic lung conditions: longitudinal observational study. JMIR Mhealth Uhealth 2020; 8: e16158.

40 Moor CC, Wapenaar M, Miedema JR, et al. A home monitoring program including real-time wireless home spirometry in idiopathic pulmonary fibrosis: a pilot study on experiences and barriers. Respir Res 2018; 19: 105.

41 Broos CE, Wapenaar M, Looman CWN, et al. Daily home spirometry to detect early steroid treatment effects in newly treated pulmonary sarcoidosis. Eur Respir J 2018; 51: 1702089.

42 Moor CC, van Leuven SI, Wijsenbeek MS, et al. Feasibility of online home spirometry in systemic sclerosis-associated interstitial lung disease: a pilot study. Rheumatology (Oxford) 2020; 60: 2467-2471.

43 Noth I, Cottin V, Chaudhuri N, et al. Home spirometry in patients with idiopathic pulmonary fibrosis: data from the INMARK trial. Eur Respir J 2021; 58: 2001518.

44 Moor K, Visser L, Aerts J, et al. Diurnal variation in forced vital capacity in patients with fibrotic interstitial lung disease using home spirometry: the DIVA study. Eur Respir J 2019; 54: Suppl. 63, PA2246.

45 Moor CC, Gür-Demirel Y, Wijsenbeek MS. Feasibility of a comprehensive home monitoring program for sarcoidosis. J Pers Med 2019; 9: 23.

46 Moor CC, van den Berg CAL, Visser LS, et al. Diurnal variation in forced vital capacity in patients with fibrotic interstitial lung disease using home spirometry. ERJ Open Res 2020; 6: 00054-2020.

47 Martinez FJ, Flaherty K. Pulmonary function testing in idiopathic interstitial pneumonias. Proc Am Thorac Soc 2006; 3: 315-321.

48 du Bois RM, Nathan SD, Richeldi L, et al. Idiopathic pulmonary fibrosis: lung function is a clinically meaningful endpoint for phase III trials. Am J Respir Crit Care Med 2012; 186: 712-715.

49 Erbes R, Schaberg T, Loddenkemper R. Lung function tests in patients with idiopathic pulmonary fibrosis: are they helpful for predicting outcome? Chest 1997; 111: 51-57.

50 Maher TM, Corte TJ, Kreuter M, et al. Home spirometry as a primary endpoint in clinical trials: sensitivity analyses of a randomized controlled trial of pirfenidone in patients with unclassifiable interstitial lung disease (UILD). Am J Respir Crit Care Med 2020; 201: A2575.

51 du Bois RM, Weycker D, Albera C, et al. Ascertainment of individual risk of mortality for patients with idiopathic pulmonary fibrosis. Am J Respir Crit Care Med 2011; 184: 459-466.

52 Bois R, Weycker D, Albera C, et al. Forced vital capacity in patients with idiopathic pulmonary fibrosis. Am J Respir Crit Care Med 2011; 184: 1382-1389.

53 Collard HR, King TE, Bartelson BB, et al. Changes in clinical and physiologic variables predict survival in idiopathic pulmonary fibrosis. Am J Respir Crit Care Med 2003; 168: 538-542.

54 Zappala CJ, Latsi PI, Nicholson AG, et al. Marginal decline in forced vital capacity is associated with a poor outcome in idiopathic pulmonary fibrosis. Eur Respir J 2010; 35: 830-836.

55 Flaherty KR, Andrei A-C, Murray S, et al. Idiopathic pulmonary fibrosis: prognostic value of changes in physiology and sixminutewalk test. Am J Respir Crit Care Med 2006; 174: 803-809.

56 Wijsenbeek M, Bendstrup E, Valenzuela C, et al. Design of a study assessing disease behaviour during the peri-diagnostic period in patients with interstitial lung disease: the STARLINER Study. Adv Ther 2019; 36: 232-243.

57 Hurst JR, Vestbo J, Anzueto A, et al. Susceptibility to exacerbation in chronic obstructive pulmonary disease. N Engl J Med 2010; 363: 1128-1138.

58 Swigris JJ, Swick J, Wamboldt FS, et al. Heart rate recovery after 6-min walk test predicts survival in patients with idiopathic pulmonary fibrosis. Chest 2009; 136: 841-848.

59 Seemungal TAR, Donaldson GC, Paul EA, et al. Effect of exacerbation on quality of life in patients with chronic obstructive pulmonary disease. Am J Respir Crit Care Med 1998; 157: 1418-1422.

60 Shah SA, Velardo C, Farmer A, et al. Exacerbations in chronic obstructive pulmonary disease: identification and prediction using a digital health system. J Med Internet Res 2017; 19: e69.

61 Wu W, Jordan S, Becker MO, et al. Prediction of progression of interstitial lung disease in patients with systemic sclerosis: the SPAR model. Ann Rheum Dis 2018; 77: 1326-1332.

62 Bahmer T, Kirsten AM, Waschki B, et al. Clinical correlates of reduced physical activity in idiopathic pulmonary fibrosis. Respiration 2016; 91: 497-502.

63 Wallaert B, Monge E, Le Rouzic O, et al. Physical activity in daily life of patients with fibrotic idiopathic interstitial pneumonia. Chest 2013; 144: 1652-1658.

64 Root ED, Graney B, Baird S, et al. Physical activity and activity space in patients with pulmonary fibrosis not prescribed supplemental oxygen. BMC Pulm Med 2017; 17: 154.

65 Bahmer T, Kirsten A-M, Waschki B, et al. Prognosis and longitudinal changes of physical activity in idiopathic pulmonary fibrosis. BMC Pulm Med 2017; 17: 104.

66 Jha S, Topol EJ. Adapting to artificial intelligence: radiologists and pathologists as information specialists. JAMA 2016; 316: 2353-2354.

67 Dilsizian SE, Siegel EL. Artificial intelligence in medicine and cardiac imaging: harnessing big data and advanced computing to provide personalized medical diagnosis and treatment. Curr Cardiol Rep 2014; 16: 441.

68 Neill DB. Using artificial intelligence to improve hospital inpatient care. IEEE Intell Syst 2013; 28: 92-95. 
70 Topalovic M, Das N, Burgel P-R, et al. Artificial intelligence outperforms pulmonologists in the interpretation of pulmonary function tests. Eur Respir J 2019; 53: 1801660.

71 Sanchez-Morillo D, Fernandez-Granero MA, Leon-Jimenez A. Use of predictive algorithms in-home monitoring of chronic obstructive pulmonary disease and asthma: a systematic review. Chron Respir Dis 2016; 13: 264-283.

72 Finkelstein J, Jeong IC. Machine learning approaches to personalize early prediction of asthma exacerbations. Ann N Y Acad Sci 2017; 1387: 153-165.

73 Orchard P, Agakova A, Pinnock H, et al. Improving prediction of risk of hospital admission in chronic obstructive pulmonary disease: application of machine learning to telemonitoring data. J Med Internet Res 2018; 20: e263.

74 Darcy AM, Louie AK, Roberts LW. Machine learning and the profession of medicine. JAMA 2016; 315: 551-552.

75 Janson C, Johansson G, Larsson K, et al. Use of Machine learning to predict asthma exacerbations. Eur Respir J 2020; 56: Suppl. 64, 4802.

76 Peng J, Chen C, Zhou M, et al. A machine-learning approach to forecast aggravation risk in patients with acute exacerbation of chronic obstructive pulmonary disease with clinical indicators. Sci Rep 2020; 10 : 3118.

77 Young AL, Bragman FJS, Rangelov B, et al. Disease progression modeling in chronic obstructive pulmonary disease. Am J Respir Crit Care Med 2019; 201: 294-302.

78 Noth I, Maher TM, Johannson KA, et al. Adherence to home spirometry among patients with IPF: results from the INMARK trial. Eur Respir J 2019; 54: Suppl. 63, PA2241.

79 Velardo C, Shah SA, Gibson O, et al. Digital health system for personalised COPD long-term management. BMC Med Inform Decis Mak 2017; 17: 19.

80 Swigris J, Nathan S, Tighe R, et al. STARMAP: an observational study to assess disease-relevant outcomes using home-monitoring devices in patients with idiopathic pulmonary fibrosis (IPF). Eur Respir J 2019; 54: Suppl. 63, PA1333.

81 Lenferink A, Brusse-Keizer M, van der Valk P, et al. Self-management interventions including action plans for exacerbations versus usual care in patients with chronic obstructive pulmonary disease. Cochrane Database Syst Rev 2017; 8: CD011682.

82 Wijsenbeek MS, Bendstrup E, Valenzuela C, et al. Interim analysis of patients with ILD enrolled in the STARLINER study. Eur Respir J 2019; 54: Suppl. 63, PA1335.

83 Nakshbandi G, Moor CC, Johannson KA, et al. Worldwide experiences and opinions of healthcare providers on eHealth for patients with interstitial lung diseases in the COVID-19 era. ERJ Open Res 2021; 7: 00405-2021.

84 ClinicalTrials.gov Identifier NCT04304898. An International Patient-led Registry in Fibrotic Interstitial Lung Diseases Using eHealth Technology (I-FILE). https://clinicaltrials.gov/ct2/show/NCT04304898

85 ClinicalTrials.gov Identifier NCT03979430. Early Detection of Acute Exacerbation in Patients with Idiopathic Lung Fibrosis - a Pilot Study. https://clinicaltrials.gov/ct2/show/NCT03979430

86 Ranjan Y, Althobiani M, Jacob J, et al. Remote assessment of lung disease and impact on physical and mental health (RALPMH): protocol for a prospective observational study. JMIR Res Protoc 2021; 10 : e28873. 